\title{
Acknowledgment of Reviewers, 2003
}

The success of Phytopathology depends on the quality of manuscripts submitted by authors and on the care and competence with which they are reviewed. It is the policy of the Editorial Board to solicit reviews for manuscripts from specialists who are most qualified to review them. In addition to members of the Editorial Board, the individuals listed below provide constructive critical reviews of one or more manuscripts during the past year. Their names are published here in grateful appreciation of their contribution to the journal and to the science of phytopathology. Publication no. P-2003-1115-01O

Anderson, A., Utah State University, Logan, UT

Adams, M., Rothamsted, Harpenden, Herts, UNITED KINGDOM

Adaskaveg, J., University of California, Riverside, CA

Adkins, S., USDA-ARS, USHR, Fort Pierce, FL

Allen, C., University of Wisconsin, Madison, WI

Alverez, A., University of Hawaii, Honolulu, HA

Anderson, J., USDA-ARS, Purdue, West Lafayette, IN

Andersen, P., NFREC, University of Florida, Quincy, FL

Aylor, D., Connecticut Agricultural Experiment Station, New Haven, CT

Bai, G., USDA-ARS, Kansas State University, Manhattan, KS

Baker, C. J., USDA-ARS, Beltsville, MD

Bakkeren, G., Ag Canada, Summerland BC, CANADA

Barash, I., Tel-Aviv University, Tel-Aviv, ISRAEL

Barbara, D., Horticulture Research International, Warwickshire, UNITED KINGDOM

Bartz, J., University of Florida, Gainesville, FL

Beattie, G. A., Iowa State University, Ames, IA

Belanger, R. R., Universite Laval, Quebec (Qc), CANADA

Benson, M., North Carolina State University, Raleigh, NC

Berger, R., University of Florida, Gainesville, FL

Bergmann, C., University of Georgia, Athens, GA

Bernard, E., The University of Tennessee, Knoxville, TN

Bird, D., North Carolina State University, Raleigh, NC

Boland, G., University of Guelph, Guelph, Ontario, CANADA

Boulton, M., John Innes Institute, UNITED KINGDOM

Brlansky, R., University of Florida, Lake Alfred, FL

Brown, J., John Innes Centre, Colney, Norwich, UNITED KINGDOM

Brown, J. K., University of Arizona, Tucson, AZ

Brown, S. K., Cornell University, Geneva, NY

Bruton, B., USDA-ARS, Lane, OK

Buchala, A., Albert-Gockel University, SWITZERLAND

Buck, K., Imperial College of Science, Technology and Medicine, London, UNITED KINGDOM

Buck, J., University of Georgia, Griffin, GA

Bull, C. T., USDA-ARS, Salinas, CA

Carbone, I., North Carolina State University, Raleigh, NC

Carisse, O., Agric \& Agri-Food Canada, St-Jean-Sur-Richelieu, CANADA

Carta, L., USDA-ARS, PSI, BARC-W, Beltsville, MD

Castlebury, L., USDA ARS SBML, Beltsville, MD

Castoria, R., Universita del Molise, Campbasso, ITALY

Cervone, F., University La Sapienza, Rome, ITALY

Chakraborty, S., University of Queensland, Brisbane, AUSTRALIA

Chang, C.-J., University of Georgia, Griffin, GA

Chappell, J., University of Kentucky, Lexington, KY

Chatterjee, A., University of Missouri-Columbia, Columbia, MO

Chellemi, D. O., USDA-ARS, Ft. Pierce, FL

Chen, J., USDA-ARS, Fresno, CA

Chen, X., USDA-ARS, Pullman, WA

Chiarappa, L., University of California, Davis, CA

Chun, W., University of Idaho, Moscow, ID

Chung, K.-R., University of Florida, Lake Alfred, FL

Clark, C., Louisiana State University, Baton Rouge, LA

Clough, S., USDA, Urbana, IL

Collmer, A., Cornell University, Ithaca, NY

Cook, D., University of California, Davis, CA

Cooksey, D., University of California, Riverside, CA

Copes, W., USDA-ARS, Poplarville, MS

Cooper, R. M., University of Bath, UK

Coplin, D., Ohio State University, Columbus, $\mathrm{OH}$

Correa, F., CIAT, Cali, Columbia
Cowger, C., Oregon State University, Corvallis, OR

Cox, K., University of Georgia, Athens, GA

Dandekar, A., University of California, Davis, CA

Davelos, A., University of Minnesota, St. Paul, MN

Davis, J., University of Florida, Gainesville, FL

de Boer, W., Netherlands Institute of Ecology, Heteren, THE NETHERLANDS

De Boer, S., Canadian Food Inspection Agency, Charlottetown, Prince Edward Island, CANADA

De Ley, I. T., University of California, Riverside, CA

Denison, S., Eckerd College, St. Petersburg, FL

Denny, T., University of Georgia, Athens, GA

Dewey, M., University of California, Davis, CA

De Wolf, E., The Pennsylvania State University, University Park, PA

Di Giovanni, G., Texas Agricultural Experiment Station, El Paso, TX

Dorrance, A., Ohio State University, Wooster, $\mathrm{OH}$

Douhan, G., University of California, Davis, CA

Duffy, B., Swiss Federal Research Station Wadenswil CH-8820, SWITZERLAND

Ebbole, D., Texas A\&M University, College Station, TX

Elliott, M., University of Florida, Fort Lauderdale, FL

Elmer, W., The Connecticut Agricultural Experiment Station, New Haven, CT

English, J., University of Missouri, Columbia, MO

Esker, P., Iowa State University, Ames, IA

Falk, B., University of California, Davis, CA

Faris, J., North Dakota State University, Fargo, ND

Farman, M., University of Kentucky, Lexington, KY

Fegan, M., The University of Queensland, Queensland, AUSTRALIA

Ferrandino, F. J., Connecticut Agric. Exp. Station, New Haven, CT

Finckh, M., University of Kassel, GERMANY

Fjellstrom, R. G., USDA-ARS, Beaumont, TX

Flier, W., IPO-DLO, Wageningen, THE NETHERLANDS

Forester, H., University of California, Riverside, CA

Franc, G., University of Wyoming, Laramie, WY

Francl, L., Penn State University, University Park, PA

Fravel, D., USDA-ARS, Beltsville, MD

Freeman, S., The Volcani Center, Bet Dagan, ISRAEL

French, R., USDA-ARS, Lincoln, NE

Fry, W., Cornell University, Ithaca, NY

Gabriel, D., University of Florida, Gainesville, FL

Gadoury, D., Cornell University, Geneva, NY

Gage, D., University of Connecticut, Storrs, CT

Garcia, J. A., Centro Nacional de Biotecnologia, Campus de la, Universidad Autonomal, MADRID, SPAIN

Garcia-Arenal, F., Unversidad Politecnica de Madrid, Madrid, SPAIN

Geider, K., Max-Planck-Institut fur Zellbiologie, Ladenburg, GERMANY

Gibb, K., Charles Darwin University, Darwin, AUSTRALIA

Gilbertson, R. L., University of California, Davis, CA

Gonsalves, D., USDA-ARS PWA, Hilo, HI

Goodell, P., University of California, Parlier, CA

Goodin, M., University of Kentucky, Lexington, KY

Goodman, B. A., The Scottish Crop Research Institute, Invergowrie, Dundee, DD2 5DA, Scotland, UK

Goodwin, S., USDA-ARS, Purdue University, West Lafayette, IN

Goodwin, S. B., USDA-ARS, West Lafayette, IN

Gordon, T., University of California, Davis, CA

Gray, S., USDA-ARS, Cornell University, Ithaca, NY

Grove, G. G., Washington State University, Prosser, WA

Grube, R., USDA-ARS, Salinas, CA 
Grunwald, N., USDA-ARS, Prosser, WA

Guan, J., Iowa State University, Ames, IA

Gubler, W. D., University of California, Davis, CA

Gulya, T., USDA-NCSL, Fargo, ND

Guo, B., USDA-ARS, Tifton, GA

Hammerschmidt, R., Michigan State University, East Lansing, MI

Hansen, E., Oregon State University, Corvallis, OR

Hanson, S., New Mexico State University, Las Cruces, NM

Harman, G., Cornell University, Geneva, NY

Harrington, R., Rothamsted Research Station, UNITED KINGDOM

Harrison, N. A., University of Florida, Ft. Lauderdale, FL

Hartung, J., USDA-ARS FL, Beltsville, MD

Havey, M. J., USDA-ARS Madison, WI

Hein, G., University of Nebraska, Scottsbluff, NE

Helgeson, J. P., University of Wisconsin, Madison, WI

Herrera-Estrella, A., CINVESTAV Irapuato, MEXICO

Hiemstra, J. A., Plant Research International BV, Wageningen, THE NETHERLANDS

Hillman, B., Rutgers University, New Brunswick, NJ

Hogenhout, S., Ohio State University, Wooster, OH

Hohn, T., FMI, Basel, SWITZERLAND

Hoitink, H., Ohio State University, Wooster, OH

Hong, C., VPI \& State University, Virginia Beach, VA

Hood, I., Forest Resource Institute, NEW ZEALAND

Hopkins, D. L., University of Florida, Apopka, FL

Horsley, R. D., North Dakota State University, Fargo, ND

Howell, C., USDA-ARS, College Station, TX

Hoy, J., Louisiana St. University, Baton Rouge, LA

Hrazdina, G., Cornell University, Geneva, NY

Hu, S., North Carolina State University, Raleigh, NC

Hughes, G., University of Edinburgh, Scotland, UNITED KINGDOM

Hulbert, S., Kansas State University, Manhattan, KS

Humber, R., USDA-ARS Collection of Entomopathogenic Fungal Cultures (ARSEF), Ithaca, NY

Hunger, R., Oklahoma State University, Stillwater, OK

Ishimaru, C., Colorado State University, Fort Collins, CO

Jabaji-Hare, S., McGill University, MacDonald Campus, St. Anne de Bellevue, CANADA

Jacobs, K., The Morton Arboretum, Lisle, IL

Jacobsen, B., Montana State University, Bozeman, MT

Jaffee, Br., University of California, Davis, CA

Janisiewicz, W., USDA-ARS Appalachian Fruit Research Station, Kearneysville, WV

Jeffers, S., Clemson University, Clemson, SC

Jia, Y., USDA-ARS, Stuttgart, AR

Jin, Y., South Dakota State University, Brookings, SD

Johnson, K., Oregon State University, Corvallis, OR

Jones, R. K., University of Minnesota, St. Paul, MN

Judelson, H., University of California, Riverside, CA

Jurjevic, Z., University of Georgia, Tifton, GA

Kado, C., University of California, Davis, CA

Kamoun, S., Ohio State University, Wooster, OH

Kang, S., The Pennsylvania State University, University Park, PA

Kast, W., Staatliche Lehr und Versuchsanstalt fuer Wein und Obstbau Weinsberg

Keller, M., Washington State University, Prosser, WA

Kelly, J. D., Michigan State University, East Lansing, MI

Kema, G., Plant Research International, Wageningen, THE NETHERLANDS

Kenerley, C., Texas A\&M, College Station, TX

Kenyon, D., Natl. Inst. of Agric. Botany, Cambridge, UNITED KINGDOM

Khush, G. S., Davis, CA

Kirk, W., Michigan State University, East Lansing, MI

Kiss, L., Hungarian Academy of Sciences, Budapest, HUNGARY

Kistler, H. C., USDA-ARS, University of Minnesota, St. Paul, MN

Kitajima, E., University of Sao Paolo, BRAZIL

Kolb, F. L., University of Illinois at Urbana-Champaign, Urbana, IL

Kolmer, J., USDA-ARS, University of Minnesota, St. Paul, MN

Kolomiets, M., Texas A\&M University, College Station, TX

Korban, S. S., University of Illinois, Urbana, IL

Kurle, J., University of Minnesota, St. Paul, MN

Lane, L., University of Nebraska, Lincoln, NE

Lannou, C., INRA, Thiverval-Grignon, FRANCE

Lazarovits, G., Agric. \& Agri-Food Canada, London, ON, CANADA
Lee, I.-M., USDA-ARS, BARC, Beltsville, MD

Lee, R., University of Florida, Lake Alfred, FL

Legard, D., California Strawberry Commission, Watsonville, CA

Legendre, L., University of Western Sydney, Penrith South DC, NSW, AUSTRALIA

Leonard, K., University of Minnesota, St. Paul, MN

Leslie, J., Kansas State University, Manhattan, KS

Lightfoot, D., Southern Illinois University, Carbondale, IL

Lopez, M., IVIA-Inst. Valenciano De Invest Agrarias, Moncada, Valencia, SPAIN

Lorita, M., University Degli Studi Di Napoli, Portici, ITALY

Luo, Y., University of California, Davis, CA

Madden, L. V., Ohio State University, Wooster, $\mathrm{OH}$

Mahaffee, W., USDA-ARS Horticulture Crops Research Lab, Corvallis, OR

Manning, W., University of Massachusetts, Amherst, MA

Marco, Y., CNRS INRA UMR, Castanet, Tolosan, FRANCE

Marek, S., University of California, Davis, CA

Marois, J., University of Florida, Quincy, FL

Marshall, D., Texas A\&M University, Dallas, TX

Martin, S. B., Pee Dee Research \& Education Center, Florence, SC

Matheron, M., University of Arizona, Yuma, AZ

Mavrodi, O. V., Washington State University, Pullman, WA

Mazzola, M., USDA-ARS, Wenatchee, WA

McDonald, B., ETH Zentrum, Zurich, SWITZERLAND

McEachrin, M., University of Georgia, Athens, GA

McMullen, M., North Dakota State University, Fargo, ND

McRoberts, N., Scottish Agricultural College, Ayr, UNITED KINGDOM

McSpadden, B. B., Ohio State University, Wooster, OH

Melcher, U., Oklahoma State University, Stillwater, OK

Menge, J., University of California, Riverside, CA

Meyer, S. L., USDA-ARS, Beltsville, MD

Ngugi, H., University of Georgia, Athens, GA

Michailides, T. J., University of California, Parlier, CA

Milgroom, M., Universidad Politécnica de Madrid, Madrid, SPAIN

Miller, W. A., Iowa State University, Ames, IA

Mims, C., University of Georgia, Athens, GA

Mirkov, T. E., Texas Agricultural Experiment Station, Weslaco, TX

Mitchell, T., North Carolina State University, Raleigh, NC

Moorman, G., Penn State University, University Park, PA

Morris, T. J., University of Nebraska, Lincoln, NE

Mugniery, D., Inst. Natl. de la Recherche Agric., Le Rheu Cedex, FRANCE

Nagy, P., University of Kentucky, Lexington, KY

Neat, S., North Dakota State University, Fargo, ND

Nelson, R. L., Cornell University, Ithaca, NY

Nelson, L., University of Saskatchewan, Saskatchewan, CANADA

Newton, A., Scottish Crop Research Institute, Invergowrie, Scotland, UNITED KINGDOM

Ngugi, H., University of Georgia, Athens, GA

Noe, J. P., University of Georgia, Athens, GA

Norelli, J., USDA-ARS, USDA-ARS, Appalachian Fruit Research Station, Kearneysville, WV

O'Donnell, K., USDA-ARS, Peoria, IL

Olanya, M., USDA-ARS, Orono, ME

Osbourn, A., Sainsbury Lab, UNITED KINGDOM

Packer, L., University of Southern California, Los Angeles, CA

Palukaitis, P., Scottish Crop Research Institute, Invergowrie, Dundee, Scotland, UNITED KINGDOM

Panella, L., USDA-ARS, Fort Collins, CO

Panopoulos, N., Inst. of Molecular Biology \& Biotechnology, Heraklion, Crete, GREECE

Paulitz, T., USDA-ARS, Washington State University, Pullman, WA

Payne, G. A., North Carolina State University, Raleigh, NC

Pethybridge, S., University of Tasmania, Burnie, TAS, AUSTRALIA

Perry, K., Cornell University, Ithaca, NY

Perry, R., IACR Rothamsted, Harpen Herts, UK

Peters, D., Wageningen University, Wageningen, THE NETHERLANDS

Pietravalle, S., Institute of Arable Crop Research, Hertfordshire, UNITED KINGDOM

Pinkerton, J., USDA-ARS, HCRL, Corvallis, OR

Roossinck, M., The Noble Foundation, Ardmore, OK

Powell, A., University of California, Davis, CA

Press, C. M., USDA-ARS, Corvallis, OR 
Prusky, D., The Volcani Center, Bet-Dagan, ISRAEL Pruvost, O., CIRAD, Reunion Island, FRANCE

Pryor, B., University of Arizona, Tucson, AZ

Punja, Z., Simon Fraser University, Burnaby, BC, CANADA

Purcell, A. H., University of California, Berkeley, CA

Qiu, W., Southwest Missouri State University, Moutain Grove, MO

Rabenstein, F., Federal Center for Breeding Research on Cultivated

Plants, Institute for Resistance Research and Pathogen Diagnostics, Aschersleben, GERMANY

Redinbaugh, M., USDA-ARS, Wooster, OH

Rezende, J. A. M., University of Sao Paolo, Piracicaba, BRAZIL

Rines, H. W., University of Minnesota, St. Paul, MN

Riley, M. B., Clemson University, Clemson, SC

Ritchie, D., North Carolina State University, Raleigh, NC

Rivera-Bustamante, R., Cinvestav Inapuato, Irapuato, Gto, MEXICO

Roberts, D., USDA-ARS, Sustainable Agricultural Systems Lab, Beltsville, MD

Rollins, J., University of Florida, Gainesville, FL

Ronald, P., University of California, Davis, CA

Rush, C., Texas A\&M Agric. Exp. Station, Bushland, TX

Sanogo, S., New Mexico State University, Las Cruces, NM

Schaad, N., USDA-ARS, Fort Detrick, MD

Schmitt, D., University of Hawaii-Manoa, Honolulu, HI

Schnabel, G., Clemson University, Clemson, SC

Schneider, W., USDA-ARS, Fort Dietrick, MD

Schottel, J., University of Minnesota, St. Paul, MN

Schumann, E., Institut für Pflanzenzüchtung und Pflanzenschutz, Halle, GERMANY

Seemueller, E., Biologische Bundesanstalt, Institut fuer Pflanzenschutz im Obstbau, Dossenheim, GERMANY

Shah, D. A., STA Laboratories, Inc., Longmont, CO

Shaner, G., Purdue University, West Lafayette, IN

Shank, R., USDA-ARS, Beaumont, TX

Shapiro, A., University of Delaware, Newark, DE

Shaw, D., Siambra Gwynion, Bangor, Gwynedd, UNITED KINGDOM

Shaw, M., University of Reading, Reading, UNITED KINGDOM

Sherwood, J., University of Georgia, Athens, GA

Shew, H. D., North Carolina State University, Raleigh, NC

Shoda, M., Tokyo Institute of Technology, Yokohama, JAPAN

Simcox, K., Pioneer Hi-Bred, International, Inc., Johnston, IA

Simpson, J., Cinvestav-IPN, Irapuato, Gto., MEXICO

Sims, J., University of California, Riverside, CA

Singh, S., University of Idaho, Kimberly, ID

Singh, S., Kansas State University, Manhattan, KS

Sipes, B. S., University of Hawaii, Honolulu, HI

Slippers, B., University of Pretoria, Pretoria, SOUTH AFRICA

Smilanick, J., USDA-ARS, Parlier, CA

Snetselaar, K., St. Josephs University, Philadelphia, PA

Spotts, R., Mid-Columbia Agric. Res. \& Ext. Center, Hood Rive, OR

Staples, R., Cornell University, Ithaca, NY

Starr, J. L., Texas A\&M University, College Station, TX

Steadman, J., University of Nebraska, Lincoln, NE

Steck, T. R., University of North Carolina at Charlotte, Charlotte, NC
Steddom, K., Texas Agric Experiment Station, Bushland, TX

Steffenson, B., University of Minnesota, St. Paul, MN

Stenlid, J., Institutionen för skoglig mykologi och patologi Sveriges lantbruksuniversitet, Uppsala, SWEDEN

Stotz, H., Oregon State University, Corvallis, OR

Stromberg, E., Virginia Polytechnic University, Blacksburg, VA

Subbarao, K., University of California, Davis, CA

Subramaniam, R., University of North Carolina, Chapel Hill, NC

Sundin, G. W., Michigan State University, East Lansing, MI

Sunter, G., University of Texas, San Antonio, TX

Szabo, L., University of Minnesota, St. Paul, MN

Tavantzis, S., University of Maine, Orono, ME

Tekauz, A., Agric. \& Agri-Food Canada, Winnipeg, CANADA

Timmer, L. W., University of Florida, Lake Alfred, FL

Tolin, S. A., Virginia Polytechnic Institute \& State University, Blacksburg, VA

Turechek, W. W., Cornell University-NYSAES, Geneva, NY

Tweddell, R., Université Laval, Québec, CANADA

Tyler, B., Virginia Polytechnic Institute and State University, Blacksburgh, VA

Tylka, G., Iowa State University, Ames, IA

Uchida, J., University of Hawaii, Honolulu, HI

Uddin, W., The Pennsylvania State University, University Park, PA

Upchurch, R. G., North Carolina State University, Raleigh, NC

Vaillancourt, L., University of Kentucky, Lexington, KY

Van Loon, L. C., Utrecht University, Utrecht, THE NETHERLANDS

Vleeshouwers, V. G. A. A., Wageningen University, Wageningen, THE NETHERLANDS

Vogel, J., USDA-ARS, Albany, CA

Walcott, R., University of Georgia, Athens, GA

Wang, G.-L., Ohio State University, Columbus, $\mathrm{OH}$

Weiland, J. J., USDA-ARS, Fargo, ND

Weller, D., USDA-ARS, Washington State University, Pullman, WA

Westphal, A., Purdue University, West Lafayette, IN

Wharton, P., Michigan State University, East Lansing, MI

Wheatley, R., Scottish Crop Research Institute, Dundee, UNITED KINGDOM

Wheeler, T., Texas Agric Experiment Station, Lubbock, TX

White, F., Kansas State University, Manhattan, KS

Whitham, S., Iowa State University, Ames, IA

Wilson, C. L., USDA-ARS, Appalachian Fruit Res., Kearneysville, WV

Wilson, M., The Colorado College, Colorado Springs, CO

Workneh, F., Texas A\&M University, Bushland, TX

Wright, S. A., Goteborg University, Goteborg, SWEDEN

$\mathrm{Wu}, \mathrm{B}$. M., University of California, Salinas, CA

Yang, B., Kansas State University, Manhattan, KS

Yang, Y., University of Arkansas, Fayetteville, AR

Yokomi, R. K., USDA-ARS PWA, Parlier, CA

Yuen, J., Swedish Agric. University, Uppsala, SWEDEN

Zambino, P., USDA Forest Service, Moscow, ID

Zeller, K., Kansas State University, Manhattan, KS

Zerbini, M., Universdade Federal de Vicosa, Vicosa, MG, BRAZIL

Zhan, J., ETH Zentrum, Zurich, SWITZERLAND 